\title{
Characterising eye movement dysfunction in Myalgic Encephalomyelitis/Chronic
}

\section{Fatigue Syndrome}

Stephen P. Badham and Claire V. Hutchinson

School of Psychology, College of Medicine, Biological Sciences and Psychology, University of Leicester, UK.

Corresponding author: Stephen P. Badham

Address: Department of Psychology, University of Warwick, Coventry, CV4 7AL, UK

Email: s.p.badham@warwick.ac.uk.

Tel: +44/0 2476528387

Fax: +44/0 2476524225

The authors have no financial relationship with the body that funded this research. The authors have full control of all primary data and agree to allow Graefe's Archive for Clinical and Experimental Ophthalmology to review their data upon request. 


\begin{abstract}
Background People who suffer from Myalgic Encephalomyelitis/Chronic Fatigue Syndrome (ME/CFS) often report that their eye movements are sluggish and that they have difficulties tracking moving objects. However, descriptions of these visual problems are based solely on patients' self-reports of their subjective visual experiences and there is a distinct lack of empirical evidence to objectively verify their claims. This paper presents the first experimental research to objectively examine eye movements in those suffering from ME/CFS.
\end{abstract}

Methods Patients were assessed for ME/CFS symptoms and were compared to age, gender and education matched controls for their ability to generate saccades and smooth pursuit eye movements.

Results Patients and controls exhibited similar error rates and saccade latencies (response times) on prosaccade and antisaccade tasks. Patients showed relatively intact ability to accurately fixate the target (prosaccades) but were impaired when required to focus accurately in a specific position opposite the target (antisaccades). Patients were most markedly impaired when required to direct their gaze as closely as possible to a smoothly moving target (smooth pursuit).

Conclusions It is hypothesised that the effects of ME/CFS can be overcome briefly for completion of saccades, but that continuous pursuit activity (accurately tracking a moving object) even for a short time period highlights dysfunctional eye movement behaviour in ME/CFS patients. Future smooth pursuit research may elucidate and improve diagnosis of ME/CFS. 
Keywords: Myalgic Encephalomyelitis (ME), Chronic Fatigue Syndrome (CFS),eye movements, prosacades, antisaccades, smooth pursuit

\section{Introduction}

Myalgic encephalomyelitis/chronic fatigue syndrome (ME/CFS) is an acquired illness, its most salient symptoms being an increased susceptibility to fatigue, with fatigue levels often becoming severely debilitating. ME/CFS affects many individuals and its prevalence has been estimated to be between 0.2 and $0.4 \%$ of the population [1]. The illness also has widespread physiological impact and has been shown to affect the immune system [2] , the central nervous system [3], and the cardiovascular system [4]. ${ }^{1}$

There are a variety of case definitions for ME/CFS [7] and the most widely used for research is the 1994 Center for Disease Control and Prevention (CDC) definition [8]. Other case definitions for ME/CFS have been developed [5, 6], though to date none of these definitions have been formally operationalised. Diagnosing ME/CFS is based on categorising the range of symptoms reported by patients and differentiating them from the symptoms occurring with other fatigue related illnesses. It is a diagnosis of exclusion because there is currently no specific diagnostic test for ME/CFS [7]. This diagnosis can be problematic due to the number of symptoms overlapping with other illnesses and the reliance on patients to report their symptoms accurately. As such, research has begun to focus more on trying to objectively quantify the symptoms that sufferers of ME/CFS commonly report with a view to delineating the condition from other illnesses, such as depression, where there may be some symptom overlap and with which patients are commonly misdiagnosed [9].

\footnotetext{
${ }^{1}$ For comprehensive reviews of ME/CFS, see [5,6].
} 
Patients who suffer from ME/CFS often report that they require increased mental effort, relative to that required before the onset of their illness, to complete everyday activities that require cognition. Experimentally, ME/CFS patients perform poorly, relative to controls, at tasks assessing attentional and working memory capacity [10-13]. Furthermore, a number of recent neuroimaging studies have shown increased brain activation in ME/CFS patients relative to control participants during cognitive tasks [14-16]. Moreover, studies that have produced experimental evidence of cognitive problems associated with ME/CFS have demonstrated that objective measures correlate well with patients' subjective symptom reports $[10,16]$.

Fatigue-related symptoms reported by those who suffer from ME/CFS are by no means restricted to the cognitive domain. Questionnaire-based studies have revealed that patients consistently report that they experience a range of visual and vision-related problems associated with their condition. These typically include pain in the eyes, increased sensitivity to light, ghosting and double vision, difficulty in directing attention, difficulty focusing on images, slow eye movements, difficulty tracking object movement and headaches during reading [17-19]. Moreover, ME/CFS sufferers also report that the visual problems they experience often become a pervasive part of their condition, exacerbate other symptoms and affect their ability to carry out everyday tasks such as reading or driving [17-19]. Indeed, some studies report that up to $25 \%$ of those suffering from CFS reduce the frequency of driving or stop driving completely due to the visual problems they experience [18].

One of the primary visual disturbances reported by those who suffer from ME/CFS is that they experience difficulty and increased fatigue in everyday tasks that involve moving their 
eyes. In this context, they often report particular difficultly when reading, a task that involves fine eye-movements for a prolonged period of time. These and other visual symptoms could be explained by poor muscular control relating to the focus (accommodation via the ciliary muscle), light and dark sensitivity (iris contraction via the iris sphincter muscle) and movement (eye movement by the extraocular muscles) of the eye. This would correspond to motor disturbances in ME/CFS reported by [5] and ME/CFS related central nervous system dysfunction cf. [3]. Establishing the characteristics of eye movement problems in ME/CFS is therefore important because doing so may: (1) provide further understanding of the symptoms related to ME/CFS; (2) provide insights into pathophysiological impacts of the condition and (3) provide an objective measure that could be used in its diagnosis and, in particular, provide a means by which it may be distinguished from other conditions.

Despite consistent reports by ME/CFS patients of difficulty performing tasks requiring eye movements, there has been no attempt to experimentally establish the characteristics of their eye movements. The purpose of the current study was to assess the nature and extent of eye movement anomalies in ME/CFS. This is the first experimental attempt to do so. As such, we have followed the experimental parameters used previously in the schizophrenia literature [20], where eye movement analysis is common.

\section{Material and methods}

\subsection{Participants}

Twenty patients and twenty age, gender and education matched control participants participated in the study. Patients were recruited by local advertising. They were refunded travel expenses for volunteering and were offered rest breaks on a regular basis throughout 
the session. Control participants were recruited from the local community and were compensated $£ 10$ for their time. All of the patients reported that they had been given a diagnosis of ME/CFS by a medical practitioner. Before being admitted to the study, they completed the DePaul Symptom Questionnaire [21] which is a useful screening tool recently developed to assess for ME/CFS. Using this tool, it could be ascertained whether patients fulfilled the standard CDC Definition [8], the Canadian ME/CFS Case Definition [5], or the International Consensus ME Case Definition [6]. Table 1 contains a summary of patients' and control participants' details. Participants had no history of eye disease. Before the main experiment commenced, visual acuity was assessed using the Freiburg Acuity Test [22]. Normal or corrected-to-normal acuity (decimal) was within the normal range and there were no differences between patients and controls $[M=1.27, S D=0.29 ; M=1.31, S D=0.20$, respectively, $t<1]$. Ethical approval was granted by School of Psychology Ethics Committee at the University of Leicester and the National Research Ethics Service. All experimental methods adhered to the tenets of the Declaration of Helsinki.

\subsection{Apparatus, stimuli and procedure}

\subsubsection{Cognitive Speed}

All participants completed the Digit Symbol Substitution Task (DSST, [23]). The DSST is a robust measure of cognitive speed. It has been shown to relate to depression [24], and is also commonly used in cognitive aging literature to assess cognitive speed and working memory [25]. A set of digits were presented on a sheet of paper with a key that associated each digit to an abstract symbol. A grid of digits was presented on the same sheet and participants were required to fill in below each digit the corresponding symbol from the key as fast as possible for $90 \mathrm{~s}$. 


\subsubsection{Eye movement recording}

Stimuli were generated using a windows PC and presented on a $100 \mathrm{~Hz}$ CRT monitor. At the viewing distance of $70 \mathrm{~cm}, 1$ screen pixel subtended 0.0315 degrees of visual angle. Participants' heads were supported and their eye movements were recorded using the Eyelink 1000 eye tracking system (SR Research, Ontario, Canada). The gaze position of the right eye was sampled at $1000 \mathrm{~Hz}$ and the Eyelink 1000 system has $<0.01^{\circ}$ of gaze resolution (noise limited) and gaze position accuracy of $<0.5^{\circ}$. Testing took place in a dimly lit room where the monitor was the main light source. The background luminance of the monitor was approximately $51 \mathrm{~cd} / \mathrm{m}^{2}$. Measurements were taken using an LS-100 spot photometer (Konica Minolta).

A nine-point calibration was conducted before each test measurement and calibration was repeated until it was labelled as accurate by the manufacturer's software. Calibration involved fixating on a target randomly appearing in each of nine different locations spread evenly across the display. Binocular status was not measured and any differences in binocular status between groups were not therefore controlled.

\subsubsection{Prosaccades and Antisaccades}

For the prosaccade and antisaccade tasks, a central, circular fixation point was presented in black $\left(0.47^{\circ}\right.$ diameter $)$ for a minimum of $800 \mathrm{~ms}$. If the participant's eye was focused within $2.3^{\circ}$ of the fixation point then the target was allowed to appear. The target was a red circle $\left(0.63^{\circ}\right.$ diameter $)$ which appeared randomly $12^{\circ}$ to the left or right of the fixation point. The target would disappear after $1200 \mathrm{~ms}$ had passed. This was followed by a blank screen for $500 \mathrm{~ms}$ before the next trial commenced. Before each task, the eye tracker was calibrated and 
participants completed 20 practice trials. The eye tracker was then recalibrated and participants completed 40 experimental trials. For the prosaccade task, participants were instructed to look at the central fixation point and to then look at the centre of the red target when it appeared. For the antisaccade task, participants were instructed to look at the central fixation point and to then look away from the target, in the opposite direction, the same distance as the target appears from the fixation.

\subsubsection{Smooth Pursuit}

Sinusoidal pursuit stimuli replicated those previously employed by Holzman and colleagues $[20,26]$. The amplitude, frequency and number of sinusoidal target oscillations were the modal parameters of 22 studies reported in a recent review [27]. Participants were instructed to follow with their eyes the centre of a red circular target of diameter $0.63^{\circ}$ as it completed 12 sinusoidal oscillations (lasting 30s in total) horizontally across the screen. The amplitude of the oscillations was $10^{\circ}$ and the frequency was $0.4 \mathrm{~Hz}$. The target commenced from a fixed starting point $5^{\circ}$ left of the centre of the screen. The target would not begin movement until participants were fixating within $2.3^{\circ}$ of its centre. Each participant performed the smooth pursuit task three times. The eye tracker was calibrated anew before each of the three trials.

\subsection{Counterbalancing}

Participants always completed the DSST task first. The saccade tasks were always performed together with the prosaccade task before the antisaccade task. The smooth pursuit tasks were also always performed together. Counterbalancing randomly placed the saccade tasks before the smooth pursuit tasks or vice versa. Each individual patient was matched to a specific control participant with both individuals completing their tests in the same order. 


\subsection{Data analysis}

\subsubsection{Cognitive Speed (Digit Symbol Substitution Task)}

Cognitive speed was determined by the number of symbols correctly substituted within $90 \mathrm{~s}$.

\subsubsection{Prosaccades and Antisaccades}

Correct saccades were those leading to fixations in the direction of the target (for prosaccades) or in the opposite direction to the target (for antisaccades). Trials were excluded if participants did not respond within the $1200 \mathrm{~ms}$ target presentation period (less than $1 \%$ of the data). Saccade latency was measured for correct trials as the time from target onset to the time of first fixation after target onset. Positional error was calculated as the distance (number of screen pixels) between the eye gaze to the target (prosaccades) or the distance between the eye gaze to the mirror position opposite the target (antisaccades). These values were converted from pixels to degrees.

\subsubsection{Smooth Pursuit}

Maintenance gain and root mean square (RMS) distance between the gaze and target position were calculated after excluding data similarly to O'Driscol and colleagues [28]. A $650 \mathrm{~ms}$ region centred at the peak target velocity was used in the calculations. Areas of the trace excluded were the first half cycle, 200 milliseconds before and after each blink, and saccades. Saccades were defined as eye movements with acceleration greater than $3500^{\circ} / \mathrm{s}^{2}$ or eye movements with speed more than $22 \%$ faster than the on-going pursuit speed. These exclusions were also verified by visual inspection. Gain was calculated as the eye velocity divided by the target velocity and outlying values (greater than two or below zero) were excluded before calculating mean and median gain. It should be noted that the influence of 
peripheral saccade effects on gain measurements is difficult to determine from the current data. The RMS distance of the pursuit was calculated (after excluding blinks and saccades) by summing squares of the mean distance (deg) between the gaze and target position and square rooting that value. Catch up saccades (CUS) and anticipatory saccades (AS) were also calculated in accordance with the definitions described by Ross and colleagues [29]. CUS were calculated as saccades that started behind the target that reduced positional error by at least 50\%. AS were calculated as saccades that either started or finished ahead of the target, or saccades that started behind the target, were at least $1^{\circ}$, and increased positional error by at least $50 \%$.

\section{Results}

\subsection{Digit Symbol Substitution Task}

In line with the aging literature, performance correlated negatively with age $[r(20)=-.46, p$ $<.01]$, indicating that the test was conducted successfully. Importantly, there were no differences in number of symbols copied between patients and controls $[M=55.4, S D=$ 13.26; $M=60.75, S D=10.08$, respectively; $t(38)=1.44, n s]$.

\subsection{Prosaccades and antisaccades}

Figure 1 shows average (a) error-rate (failure to produce a saccade in the correct direction), (b) saccade latency (msecs) and (c) positional error (distance (deg) between eye gaze position and the desired fixation point) for ME/CFS patients and control participants when carrying out the prosaccade and antisaccade tasks. Patient and control error-rates did not differ for prosaccadic $[t(38)=1.50, n s]$ or antisaccadic $[t(38)=1.04, n s]$ eye movements (Fig 1a). 
However, saccade latency was larger (slower reactions) in patients compared to controls (Fig 1b). This effect reached marginal significance for prosaccades $\left[t\left(25.46^{2}\right)=1.77, p=.09\right]$ but not for antisaccades $[t<1]$. Patients also exhibited larger positional errors than controls. This effect reached marginal significance for prosaccades $[t(29.87)=1.94, p=.06]$ and was striking for antisaccades $[t(25.87)=3.02, p<.01]$, indicating that ME/CFS patients particularly struggled when required to direct their gaze at a specific point opposite the target when there was no object to fixate upon. Also, although there was no effect of age on the extent of positional errors for control participants, patients' antisaccade positional errors correlated positively with age $[r(20)=.53, p<.05]$, suggesting that the visual effects of ME/CFS have a greater impact with increasing age.

\subsection{Smooth pursuit}

Figure 2 shows (a) mean maintenance gain between the eye position (gaze) and the target position (eye velocity divided by the target velocity), (b) global root mean square (RMS) error, (c) catch up saccade frequency $(\mathrm{Hz})$ and $(\mathrm{d})$ anticipatory saccade frequency $(\mathrm{Hz})$ for ME/CFS patients and control participants when carrying out the smooth pursuit task across three test runs. Performance was assessed by conducting 2 (Group: patients, controls) x 3 (Test Run: $1^{\text {st }}, 2^{\text {nd }}, 3^{\text {rd }}$ ) mixed ANOVAS on each experimental measure of smooth pursuit quality. Where there was evidence of departure from the sphericity assumption, GreenhouseGeisser corrections to degrees of freedom and $p$-values are reported.

\footnotetext{
${ }^{2}$ Degrees of freedom adjusted because Levene's test for equality of variances was significant.
} 
For mean maintenance gain (Fig 2a), there was a marginal effect of group $[F(1,38)=2.98$, $M S E=0.26, p=.09$ ], with patients performing worse (exhibited lower gain) than controls. There was a also main effect of test run $[F(2,76)=3.61, M S E=0.01, p<.05]$ with performance being highest in the first, the second and then the third test runs. Although, at least numerically, performance declined more for patients than controls across the three test runs $^{3}$, there was no interaction between factors.

A clearer pattern was found with $\log _{10}$ global RMS error (Fig $2 b$ ). There was a main effect of group $[F(1,38)=4.75, M S E=2.17, p<.05]$ with patients performing significantly worse than controls, and a main effect of test run $[F(2,76)=6.49, M S E=0.08, p<.01]$ with performance being best in the first test run and deteriorating in the second and third test runs. There was also a marginal group by test run interaction $[F(2,76)=2.32, M S E=0.03, p=.11]$ with patients showing a larger drop in performance from the first test run to the second and third test runs than controls. Follow up Bonferroni adjusted (for six comparisons) paired $t$ tests confirmed this pattern of results. Patients performed significantly better in the first test run compared to the second test run $[t(19)=3.97, p<.01]$ and in the first test run compared to the third test run $[t(19)=3.31, p<.05]$. For the second test run compared to the third test run there was no difference for patients. None of the comparisons showed any differences for controls [all $t \mathrm{~s}</=1]$. This demonstrated that for the patients only, performance deteriorated as the testing session went on.

For catch up saccade (CUS) frequency (Fig 2c), although there was no main effect of group $[F<1]$, there was a main effect of test run $[F(1.47,55.92)=5.70, M S E=0.29, p<.05]$, with

\footnotetext{
${ }^{3}$ A similar pattern of results was found with the median gains averaged for each participant.
} 
poorer performance (more catch up saccades) in the first test run compared to the second and third test runs. There was no interaction between the factors.

Finally, for anticipatory saccade (AS) frequency (Fig 2d), there was no main effect of group $[F(1,38)=1.36, M S E=3.67, \mathrm{~ns}]$, although there was a main effect of test run $[F(2,76)=$ $6.62, M S E=0.68, p<.01]$, with more AS in the second test run compared to the first and third test runs. A significant interaction between the factors $[F(2,76)=4.55, M S E=0.47, p<$ $.05]$ indicated that this was driven by the patients. This was confirmed by follow up Bonferroni adjusted (for six comparisons) paired $t$-tests. For patients, there were significantly more AS in the second test run compared to the first test run $[t(19)=3.95, p<.01]$ and compared to the third test run $[t(19)=3.81, p<.01]$. There were no other differences in AS between test runs for patients or controls [all $t \mathrm{~s}<1.72]$. The AS data also showed a significant correlation with age for the patient group for the first $[r(20)=0.68 ; p<.01]$, second $[r(20)=0.53 ; p<.05]$, and third $[r(20)=0.58 ; p<.01]$ test runs (poorer performance with increasing age). This was not the case for control participants on any of the test runs and therefore indicates a greater impact of ME/CFS with increasing age.

\section{Discussion}

This study is the first to objectively assess eye-movement problems in those suffering form ME/CFS. In accordance with subjective reports, we found that patients generally performed worse than controls in tasks that required quick and accurate eye movements. Patients were only slightly impaired on tasks that required prosaccadic eye movements (those towards the target). The effects of ME/CFS on the ability to perform antisaccades (eye movements towards the hemispace opposite the target) were more pronounced, particularly for positional 
errors. The smooth pursuit task highlighted clear differences between patients and controls. Firstly, patients showed general deficits in their ability to closely track the moving target. Secondly, across the three test sessions, patients' performance deteriorated more than control participants' performance.

In the context of saccadic eye movements, patients and controls exhibited similar error rates and saccade latencies (response times). Patients also showed a relatively intact ability to accurately fixate on objects appearing within their field of view (prosaccades). The most notable area of difficulty for patients was a reduced ability to focus accurately in a specific position opposite the target (antisaccades). The key difference between the prosaccade task and the antisaccade task in this respect is that the former requires individuals to fixate on a clearly presented target, whilst the latter requires individuals to judge the fixation point themselves. As such, these findings may reflect poorer spatial awareness in patients than in controls and/or an inability to swiftly recall the exact position on which to focus when directing their gaze away from the target. Indeed, ME/CFS-related deficits in attention-based tasks have been shown previously [12]. Importantly, the presence of intact and dysfunctional performance within a single (antisaccade) task indicates a potential area in which to learn more about the selective visual/cognitive impairments related to ME/CFS.

Mean maintenance gain and $\log _{10}$ global RMS error measures of smooth pursuit quality yielded poorer performance in patients relative to controls. These smooth pursuit deficits exhibited by ME/CFS patients may represent an important diagnostic measure that could be used in future objective assessment of ME/CFS. Patients were not only poorer at the task relative to controls but this deficit increased throughout the test session. This indicates that the task was able to measure patients' increased susceptibility to the effects of fatigue on a 
short time scale (the three smooth pursuit test runs took place across approximately 5-10 minutes including time to calibrate the equipment). The smooth pursuit task required sustained musculature activity for $30 \mathrm{~s}$ and this may be a factor that differentiates it from the saccade tasks where a brief eye movement is followed by 1-2 s of inactivity. Carruthers and colleagues [5] highlight the commonality of motor disturbances and Michiels and colleagues [12] identified dysfunctional control of attention in ME/CFS patients. It may be the case that patients are able to overcome their dysfunction for brief saccade tasks but not for continuous pursuit. In this respect, future research should aim to establish which factors mediate patient deficits in smooth pursuit. If a task with large and consistent deficits can be found, then diagnosis of ME/CFS may be improved by the inclusion tasks of this type.

One, somewhat unexpected, finding was that the severity of some of deficits revealed by the eye movement measures were correlated with age for patients but not controls. The frequency of anticipatory saccades produced by patients correlated positively with age for all three test runs whilst there were no correlations for control participants. The same was found for the antisaccade positional errors, which were significantly greater with increasing age for patients but not for control participants. This suggests that the visual effects of ME/CFS are greater for older adults and indicates that the illness may be more debilitating for them, presumably because they are less able than young adults to compensate for ME/CFS related deficits. Similar results are apparent in depression where there is evidence that symptoms are more chronic in older adults compared to young adults [30].

Under the current testing conditions, we cannot rule out the possibility that blur may have contributed to our findings. The first consideration concerns older participants. We used the Freiburg Acuity Test to assess normal/corrected-to-normal visual acuity for long distance 
vision. However, at the viewing distance of $70 \mathrm{~cm}$ employed in this study, older participants may have been more prone to the effects of blur due to presbyopia. Given that patients and control participants were matched on the basis of age, it is unlikely that this would be a driving factor. The second consideration concerns ME/CFS. In this context, blur may indeed offer an explanation for our findings. Individuals with ME/CFS often report that they experience a number of symptoms related to blurred vision [18] to the extent that being 'unable to focus vision' has been incorporated into some diagnostic measures of the condition [21]. As a consequence, the red circle may have appeared more 'blurry' to patients than to controls, deleteriously affecting their ability to accurately track it as it moved across the screen. The issue of blur in ME/CFS is interesting in that it may represent an underlying factor in a number of the visual anomalies reported by those with ME/CFS and, as such, warrants further study.

In conclusion, this study was able to experimentally determine visual dysfunction in ME/CFS patients. Our findings are in good agreement with previous questionnaire based studies [1719]. It is particularly noteworthy that ME/CFS patients performed well on many aspects of the antisaccade tasks (error rate and saccade latency), yet they showed dysfunctional smooth pursuit ability. This differentiates the current pattern of results from those exhibited by patients with disorders such as schizophrenia, where patients show dysfunction on both tasks [20]. This pattern of results also supports reports by patients that they experience particular difficulty when they are required to track the movement of an object/s across visual space. Given the high of prevalence of ME/CFS across the population and common reports of visual problems associated with the condition, it is apparent that developing visual assessment tasks sensitive to ME/CFS may provide a valuable method of understanding and diagnosing the illness. 
Acknowledgements. This research was funded by ME Research UK and the Irish ME Trust. We are extremely grateful to all our participants, especially those suffering from ME/CFS for taking part in the study. We thank Professor Leonard Jason, DePaul University, Chicago for kindly providing us with the DePaul Symptom Questionnaire (DSQ). 


\section{References}

1. NICE Clinical Guideline 53, Developed by the National Collaborating Centre for Primary Care. (2007). Chronic fatigue syndrome/myalgic encephalomyelitis (or encephalopathy): Diagnosis and management of CFS/ME in adults and children. Retrieved from http://www.nice.org.uk/nicemedia/live/11824/36193/36193.pdf [Accessed 10th April 2013]

2. Lorusso, L., Mikhaylova, S.W., Capelli, E., Ferrari, D., Ngonga, G.K., Ricevuti, G. (2009). Immunological aspects of chronic fatigue syndrome. Autoimmunity Reviews, 8, 287-91.

3. Chen, R., Liang, F.X., Moriya, J., Yamakawa, J., Sumino, H., Kanda, T., \& Takahashi, T. (2008). Chronic fatigue syndrome and the central nervous system. The Journal of International Medical Research, 36, 867-874.

4. Hollingsworth, K.G., Jones, D.E., Taylor, R., Blamire, A.M., \& Newton, J.L. (2010) Impaired cardiovascular response to standing in chronic fatigue syndrome. European Journal of Clinical Investigation, 40, 608-615.

5. Carruthers, B.M., Jain A.K., De Meirleir, K.L., Peterson, D.L., Klimas, N. G., Lerner, A.M., ... van de Sande, M.I. (2003). Myalgic encephalomyelitis / chronic fatigue syndrome: Clinical working case definition, diagnostic and treatment protocols. Journal of Chronic Fatigue Syndrome. 11, 7-116.

6. Carruthers, B. M., van de Sande, M.I., .DeMeirleir, K.L., Klimas, N. G., Broderick, G., Mitchell, T., ... Stevens, S. (2011). Myalgic encephalomyelitis: International Consensus Criteria. Journal of Internal Medicine, 270, 327-338. 
7. Christley, Y., Duffy, T., \& Martin, C.R. (2012). A review of the definitional criteria for chronic fatigue syndrome. Journal of Evaluation in Clinical Practice, 18, 2531.

8. Fukuda, K., Straus, S.E., Hickie, I., Sharpe, M.C., Dobbins, J.G., \& Komaroff, A. (1994). The chronic fatigue syndrome: A comprehensive approach to its definition and study. Annals of Internal Medicine, 121, 953-959.

9. Griffith, J.P \& Zarrouf, F.A. (2008). A systematic review of chronic fatigue syndrome: Don't assume its depression. The Primary Care Companion to the Journal of Clinical Psychiatry, 10(2), 120-128.

10. Capuron, L., Welberg, L., Heim, C., Wagner, D., Solomon, L., Papanicolaou, D.A., ... Reeves, W.C. (2006). Cognitive dysfunction relates to subjective report of mental fatigue in patients with chronic fatigue syndrome. Neuropsychopharmacology, 31, 1777-1784.

11. Majer, M., Welberg, L.A.M., Capuron, L., Miller, A.H., Pagnoni, G., Reeves, W.C. (2008). Neuropsychological performance in persons with chronic fatigue syndrome.: Results from a population study. Psychosomatic Medicine, 70, 829836.

12. Michiels, V., Cluydts, R., Fischler, B. (1998). Attention and verbal learning in patients with chronic fatigue syndrome. Journal of the International Neuropsychological Society, 4, 456-66.

13. Cockshell, S.J., \& Mathias, J.L. (2010). Cognitive functioning in chronic fatigue syndrome: A meta-analysis. Psychological Medicine, 40, 1253-1267. 
14. Cook, D. B., O'Connor, P. J., Lange, G., \& Steffener, J. (2007). Functional neuroimaging correlates of mental fatigue induced by cognition among chronic fatigue syndrome patients and controls. Neuroimage, 36, 108-122.

15. Flor-Henry, P., Lind, J. C., \& Koles, Z. J. (2010). EEG source analysis of chronic fatigue syndrome. Psychiatry Research, 181, 155-164.

16. Lange, G., Steffener, J., Cook, D. B., Bly, B. M., Christodoulou, C., Liu, W.C., ... Natelson, B.H. (2005). Objective evidence of cognitive complaints in Chronic Fatigue Syndrome: A BOLD fMRI study of verbal working memory. Neuroimage, 26, 513-524.

17. Leslie, S. (1997). Chronic Fatigue Syndrome: Optometric clinical presentation and management. Journal of Behavioural Optometry, 8, 155-161.

18. Potaznick, W. \& Kozol, N. (1992). Ocular manifestations of chronic fatigue and immune dysfunction syndrome. Optometry and Vision Science, 69, 811-814.

19. Vedelago, L.J. (1997). Visual dysfunction in chronic fatigue syndrome. Journal of Behavioural Optometry, 8, 149-154.

20. Sereno, A.B., \& Holzman, P.S. (1995). Antisaccades and smooth pursuit eye movements in schizophrenia. Biological Psychiatry, 37, 394-401.

21. Jason, L.A., Evans, M., Porter, N., Brown, M., Brown. A., Hunnell, J., ... Lerch A.(2010). The development of a revised Canadian myalgic encephalomyelitis chronic fatigue syndrome case definition. American Journal of Biochemistry and Biotechnology, 6, 120-135. 
22. Bach, M. (1996) The "Freiburg Visual Acuity Test" - Automatic measurement of visual acuity. Optometry \& Vision Science, 73, 49-53

23. Wechsler, D. (1981). Manual for the Wechsler adult intelligence scale - revised. New York, NY: Psychological Corporation.

24. Austin, M.P., Mitchell, P., Wilhelm, K., Parker, G., Hickie, I., Brodaty, H., ... HadziPavlovic, D. (1999). Cognitive function in depression: A distinct pattern of frontal impairment in melancholia? Psychological Medicine, 29, 73-85.

25. Hoyer, W. J., Stawski, R. S., Wasylyshyn, C., \& Verhaeghen, P. (2004). Adult age and digit symbol substitution performance: A meta-analysis. Psychology and Aging, 19, 211-214.

26. Holzman, P.S., O’Brian, C., \& Waternaux, C. (1991). Effects of lithium treatment on eye movements. Biological Psychiatry, 29, 1001-1015.

27. Smyrnis, N. (2008). Metric issues in the study of eye movements in psychiatry. Brain and Cognition, 68, 341-358.

28. O’Driscoll, G.A, \& Callahan, B.L. (2008). Smooth pursuit in schizophrenia: A metaanalytic review of research since 1993. Brain and Cognition, 68, 359-370.

29. Ross, R.G., Olincy, A., Zerbe, G., \& Radant, A. (2003) Which duration of postsaccadic slowing identifies anticipatory saccades during smooth pursuit eye movements? Psychophysiology, 38, 325-333.

30. Bäckman, L., Small, B.J., Wahlin, A., \& Larsson, M. (2000) Cognitive Functioning in Very Old Age. In T. A. Salthouse \& F. I. M. Craik (Eds.), The handbook of aging and cognition (2nd ed., pp. 499-558). Mahwah, NJ: Lawrence Erlbaum Associates, Inc. 
Table 1. Mean (and standard deviation) age, gender and level of education of participants alongside myalgic encephalomyelitis/chronic fatigue syndrome (ME/CFS) categorisation for patients.

\section{ME/CFS Definition}

\begin{tabular}{|c|c|c|c|c|c|c|}
\hline & & & & \multirow{2}{*}{\multicolumn{2}{|c|}{$\begin{array}{ll}\text { CFS } & \text { Canadian }\end{array}$}} & \multirow{2}{*}{ International } \\
\hline Age & Age & Education $^{\mathrm{a}}$ & Gender & & & \\
\hline & & & (females/n) & (Fukuda et al., 1994). & (Carruthers et al., 2003) & (Carruthers et al., 2011) \\
\hline
\end{tabular}

\begin{tabular}{|c|c|c|c|c|c|c|c|}
\hline Patients & $44.22(13.25)$ & $24-71$ & $2.85(0.59)$ & $14 / 20$ & $20 / 20$ & $15 / 20$ & $11 / 20$ \\
\hline Controls & $44.63(13.41)$ & $24-72$ & $3.20(0.89)$ & $14 / 20$ & N/A & N/A & N/A \\
\hline Sig & $t<1$ & & $t=1.46, n s$ & & & & \\
\hline
\end{tabular}

${ }^{\mathrm{a}}$ Four levels of education: 1 = some high school (GCSE or equivalent); $2=$ partial college (A level or equivalent); $3=$ college/university degree; $4=$ graduate professional degree (masters/doctorate) 


\section{Figure Captions}

Figure 1. Prosaccade and antisaccade performance for patients and controls. a: Mean error rate (proportion). b: Saccade latency (msecs). c: Positional error (distance between the eye position and the desired fixation point). Error bars represent $\pm 1 S E$.

Figure 2. Smooth pursuit performance for patients and controls across three test runs. a: Mean maintenance gain. b: Global root mean square error. c: Catch up saccade frequency $(\mathrm{Hz})$. d: Anticipatory saccade frequency $(\mathrm{Hz})$. Error bars are $\pm 1 S E$. 
Figure 1
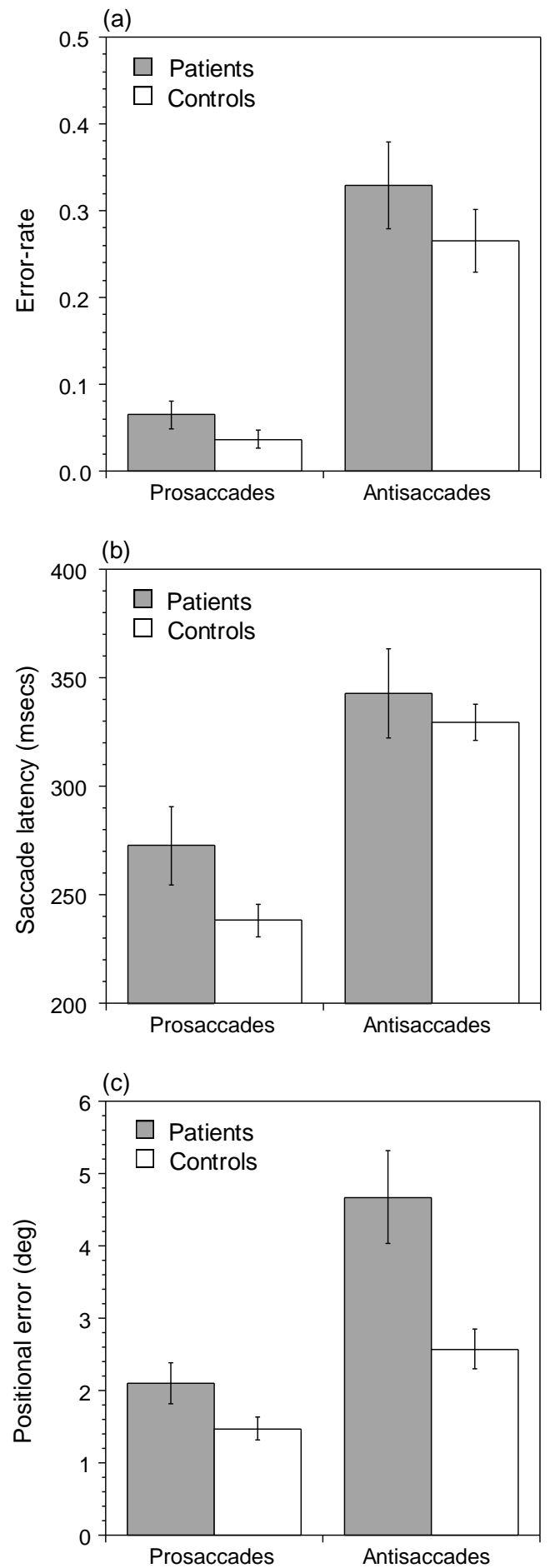
Figure 2
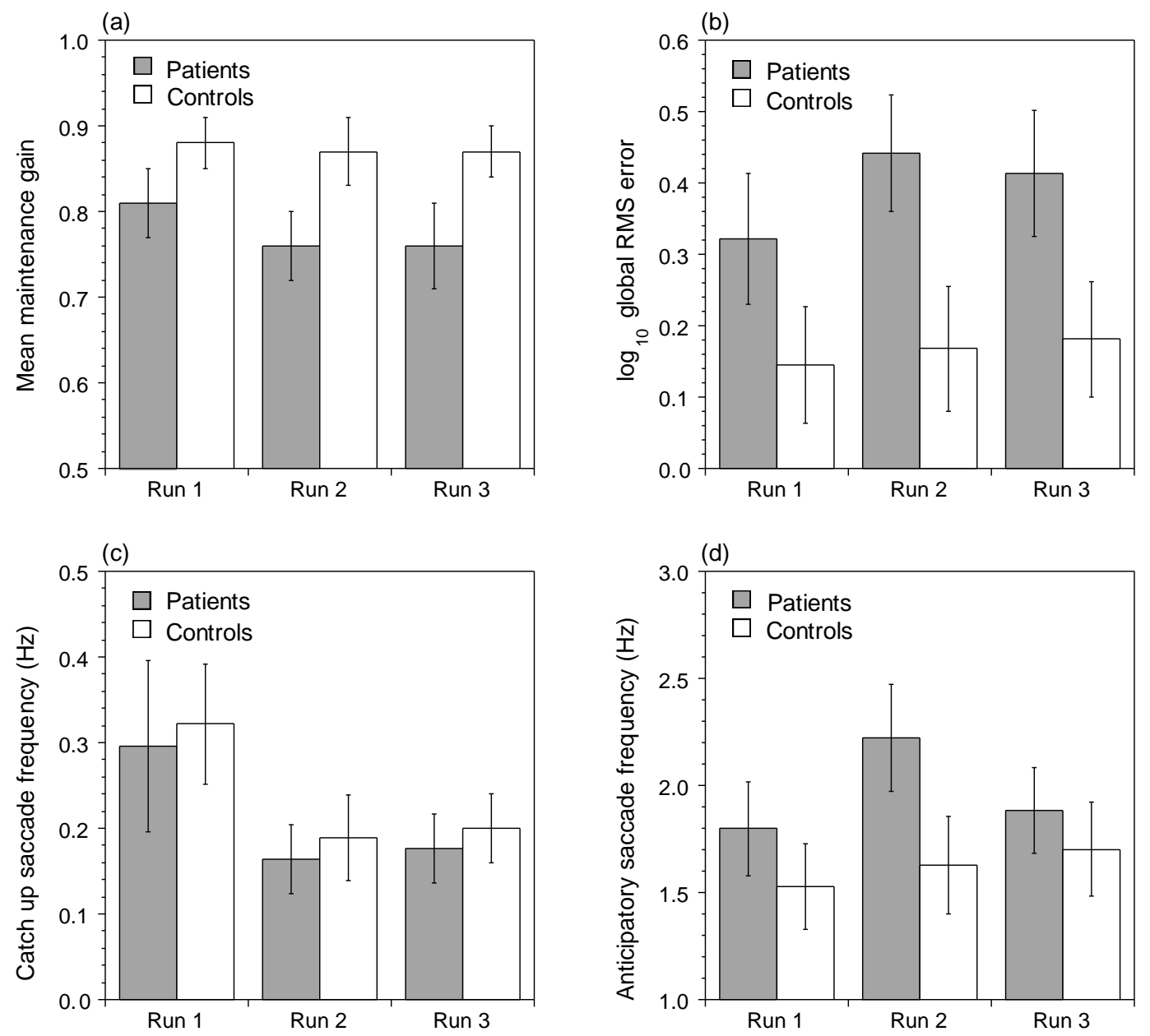